\title{
Serum-free-medium-type mesenchymal stem cell culture supernatant exerts a protective effect on A549 lung epithelial cells in acute lung injury induced by $\mathrm{H}_{2} \mathrm{O}_{2}$
}

\author{
JIAN WU $^{1 *}$, AN-QUAN SHANG ${ }^{2 *}$, CHU CHEN $^{3}$, WEI-WEI WANG ${ }^{4}$, \\ CUN-QUAN XIONG ${ }^{5}$ and NAI-ZHOU GUO ${ }^{1}$ \\ ${ }^{1}$ Laboratory of Medicine, The First People's Hospital of Yancheng City, Yancheng, Jiangsu 224005; \\ ${ }^{2}$ Department of Laboratory Medicine, Tongji Hospital of Tongji University, Shanghai 200092; \\ ${ }^{3}$ Kangda College of Nanjing Medical University, Lianyungang, Jiangsu 222000 \\ ${ }^{4}$ Department of Laboratory Medicine, The Sixth People's Hospital of Yancheng City, Yancheng, Jiangsu 224005; \\ ${ }^{5}$ College of Pharmacy, Jiangsu Vocational College Medicine, Yancheng, Jiangsu 224002, P.R. China
}

Received March 28, 2018; Accepted August 10, 2018

DOI

Abstract. The aim of the present study was to investigate the mechanisms and protective effect of serum-free-medium-type fetal placental mesenchymal stem cell (fPMSC) culture supernatant on A549 lung epithelial cells following treatment with hydrogen peroxide $\left(\mathrm{H}_{2} \mathrm{O}_{2}\right)$. A549 lung epithelial cells were stimulated with different concentrations of $\mathrm{H}_{2} \mathrm{O}_{2}$, and the survival rate of the cells was examined by Cell Counting Kit- 8 (CCK-8) assay. It was concluded that the $\mathrm{H}_{2} \mathrm{O}_{2}$ concentration when the cell survival rate was at $50 \%$ was the optimum condition to create an oxidative damage model. Hoechst 33258 staining and western blot analysis was used to validate the A549 lung epithelial cell model. Serum-free medium was used to culture fPMSCs, and A549 lung epithelial cells treated with $\mathrm{H}_{2} \mathrm{O}_{2}$ were cultured with passage 3 MSC supernatant for $24 \mathrm{~h}$. This was termed the supernatant group. Simultaneously, a damage group that was stimulated with $\mathrm{H}_{2} \mathrm{O}_{2}$ only, and a vitamin $\mathrm{C}$ (VC) group that was treated with $\mathrm{H}_{2} \mathrm{Q}_{2}$ followed by $100 \mu \mathrm{mol} / 1 \mathrm{VC}$ in eulture medium was also established. The apoptosis of the three groups was detected by flow cytometry,

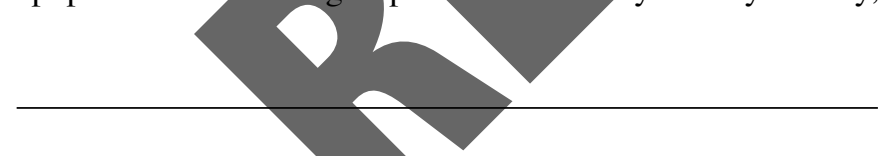

Correspondence to: Dr Cun-Quan Xiong, College of Pharmacy, Jiangsu Vocational College Medicine, 283 JieFang Road, Yancheng, Jiangsu 224002, P.R. China

E-mail: 89131245@qq.com

Dr Nai-Zhou Guo, Laboratory of Medicine, The First People's Hospital of Yancheng City, 166 Yulong Road, Yancheng, Jiangsu 224005, P.R. China

E-mail: gnz120@163.com

*Contributed equally

Key words: mesenchymal stem cells, acute lung injury, A549, lung epithelial cells and western blotting was used to detect apoptosis-associated and nuclear factor erythroid 2-like 2 (Nrf2)-kelch-like ECH-associated protein 1 (Keap1)-antioxidant response element/oxidative stress-associated protein expression. Following the CCK-8 test, $600 \mu \mathrm{mol} / 1 \mathrm{H}_{2} \mathrm{O}_{2}$ was selected to stimulate the A549 lung epithelial cells for $24 \mathrm{~h}$, which resulted in a A549 cell survival rate of $56.41 \pm 3.31 \%$. Hoechst 33258 staining and western blotting also confirmed the reliability of the model. Flow cytometry demonstrated that the apoptotic rate of the cells in the VC and supernatant groups was reduced compared with that in the injury group. The difference between the supernatant group and the injury group was statistically significant. The detection of apoptosis-associated proteins by western blotting revealed that the expression of apoptosis regulator BAX and Caspase- 3 in the VC and supernatant groups was decreased. Furthermore, the expression of B-cell lymphoma-2 was increased compared with that in the injury group, and the difference was statistically significant $(\mathrm{P}<0.05)$. Compared with that in the injury group, the expression of Nrf2 increased in the VC and supernatant groups, whereas the expression of Keap1 was decreased, and the difference was statistically significant $(\mathrm{P}<0.05)$. In conclusion, fPMSC supernatant exhibited an antioxidant capacity in A549 lung epithelial cells treated with $\mathrm{H}_{2} \mathrm{O}_{2}$ as a model of acute lung injury. The supernatant was found to reduce oxidative damage and inhibit apoptosis.

\section{Introduction}

Acute lung injury (ALI) is characterized by rapid onset lung and capillary endothelial cell damage caused by a series of factors (1), which may consequently result in pulmonary edema and hypoxic respiratory insufficiency. The clinical manifestations include progressive hypoxemia and respiratory distress. Severe ALI is termed acute respiratory distress syndrome (ARDS).

According to the literature (2), the mortality rate of ALI is $35-40 \%$. Studies investigating the mechanism of ALI 
have revealed that its etiology involves imbalances between inflammatory and anti-inflammatory factors, as well as oxidant and antioxidant capacity (3-5), indicating that oxidative stress and excessive inflammatory factor release are critical factors that should be targeted in the treatment of lung injury.

When the body is confronted by a series of external stimuli, the oxidant and antioxidant system become imbalanced, which may lead to reactive oxygen species (ROS) accumulation, resulting in oxidative damage to tissues and organs. This phenomenon is termed oxidative stress (6). It has been demonstrated that the nuclear factor erythroid 2-like 2 (Nrf2)-kelch-like ECH-associated protein 1 (Keap1)-antioxidant response element (ARE) signaling pathway is a major regulator of the antioxidant response (7).

Under normal physiological conditions, Nrf2 molecules bind to the Keap1 protein molecules in the cytoplasm and are in an inactive state, and are therefore unable to translocate to the nucleus to activate transcription. When oxidative stress occurs, Nrf2 and Keap1 uncouple, Nrf2 is phosphorylated and translocates to the nucleus to bind ARE, and the transcription of downstream detoxification enzymes and antioxidant genes is initiated, in order to increase the antioxidant ability of the cell (8).

Mesenchymal stem cells (MSCs) originate from the early developmental mesoderm and are multipotent cells; they have been recognized as an ideal seed for tissue repair due to their strong multilineage differentiation potential and immunoregulatory ability (9). Shalaby et al (10) confirmed that MSCs alleviate lung injury and improve the activity of antioxidan enzymes in serum, by injecting MSCs into the caudal vein of a rat model. In addition, the use of MSCs in the treatment of lung disease is not limited to laboratory and animal models, but has also been partially developed as a clinical treatment. Wilson et al (11) used MSCs for the clinical treatment of ARDS, and achieved successful results. Furthermore, it has been suggested that MSCs have anti-inflammatory and antioxidant effects, due to their powerful paracrine function (12). When exogenous MSCs are administered, inflammation and oxidative stimuli are reduced through the secretion of anti-inflammatory factors and antioxidant enzymes, resulting in tissue injury prevention. Therefore, it has been suggested that the supernatant of MSCs also has the ability to repair tissue (13). Our previous study confirmed that serum-free-medium-type MSC supernatant has the ability to scavenge reactive oxygen species, and its total antioxidant capacity is equivalent to $100 \mu \mathrm{mol} / 1$ vitamin C (VC) (14). Therefore, $100 \mu \mathrm{mol} / 1$ was selected as a positive control in the present study, in order to elucidate whether the supernatant of MSC culture protected and/or repaired lung epithelial cells damaged by ROS.

\section{Materials and methods}

Establishment of an oxidative damage model. A549 cells, which were maintained in $\mathrm{F} 12 \mathrm{~K}$ medium at $37^{\circ} \mathrm{C}$ in $5 \% \mathrm{CO}_{2}$, were purchased from the Chinese Academy of Sciences Cell Bank (Shanghai, China), and were cultured in high-glucose Dulbecco's modified Eagle's medium. When the cell confluence reached 80\%, TrypLE reagents (Gibco; Thermo Fisher Scientific, Inc., Waltham, MA, USA) were used to dissociate the cells in order to obtain a cell suspension. Cells were plated at a density of $5 \times 10^{3}$ cells/well in a 96 -well plate, and subsequently cultured for $12 \mathrm{~h}$ at $37^{\circ} \mathrm{C}$ in an incubator.

Next, solutions with final concentrations of 200,400 , 500,600 and $800 \mu \mathrm{mol} / 1$ hydrogen peroxide $\left(\mathrm{H}_{2} \mathrm{O}_{2}\right)$ oxidation medium were prepared and added to the wells for 6,12 or $24 \mathrm{~h}$. CCK- 8 was used to detect the survival rate of the cells. Each test was repeated five times in parallel. The optimal $\mathrm{H}_{2} \mathrm{O}_{2}$ concentration and stimulation duration for the oxidative damage model were those recorded at a cell survival rate of $50 \%$, and this concentration was used for future experiments.

Hoechst 33258 staining. The A549 cell suspension was added into 6-well plates. The experiment used two groups: The normal group (untreated cells) and the $\mathrm{H}_{2} \mathrm{O}_{2}$-induced injury group. The two groups of cells were made into separate cell slides and stained with Hoechst 33258 for $5 \mathrm{~min}$ at $37^{\circ} \mathrm{C}$. Cell damage in the two groups was observed under a fluorescence microscope.

Western blot analysis. Total protein concentration was determined using a bicinchoninic acid protein assay kit (cat. no. C503021; Sangon Biotech Co., Ltd., Shanghai, China) according to the manufacturer's protocols. Protein lysates from cells were prepared by using a radioimmunoprecipitation assay (RIPA) kit (Beyotime Institute of Biotechnology, Shanghai, China) according to the manufacturer's protocols. Equivalent amounts of protein ( $80 \mu \mathrm{g}$ per lane) were separated on $10 \%$ sodium dodecyl sulfate-polyacrylamide gel electrophoresis gels and subsequently transferred to polyvinylidene difluoride membranes (EMD Millipore, Billerica, MA, USA). The membranes were blocked in 5\% non-fat milk at room temperature for $1 \mathrm{~h}$, and then incubated with BAX (cat. no. sc-7480; 1:1,000 dilution; Santa Cruz Biotechnology, Inc., Dallas, TX, USA), Bcl-2 (cat. no. sc-7382; 1:1,000 dilution; Santa Cruz Biotechnology, Inc.), Caspase-3 (cat. no. 9661; 1:1,000 dilution; Cell Signaling Technology, Inc., Danvers, MA, USA), Nrf2 (cat. no. ab31163; 1:5,000 dilution; Abcam, Cambridge, MA, USA), Keap1 (cat. no. 10503-2-AP; 1:2,000 dilution; Proteintech Group, Inc., Chicago, IL, USA) and $\beta$-actin (cat. no. 3700S; 1:1,000 dilution; Cell Signaling Technology, Inc.) primary antibodies at $4^{\circ} \mathrm{C}$ overnight. Subsequent to being washed three times with Tris-buffered saline with Tween-20 (TBST), the membranes were probed with secondary peroxidase-conjugated antibodies (cat. nos. bs-0296R and bs-0295M; 1:1,000 dilution; Beijing Biosynthesis Biotechnology Co., Ltd., Beijing, China) at room temperature for $2 \mathrm{~h}$. Subsequent to washing the membranes three times with TBST, the binding protein was developed and fixed in the darkroom following enhanced chemiluminescence (cat. no. C510043; Sangon Biotech Co., Ltd.). Western blot analysis was performed to determine the relative expression of the target protein normalized to $\beta$-actin.

Apoptosis detection by flow cytometry. Three experimental groups were set up for flow cytometry: i) $\mathrm{H}_{2} \mathrm{O}_{2}$ stimulation only (injury group); ii) $\mathrm{H}_{2} \mathrm{O}_{2}$ stimulation followed by $100 \mathrm{~mol} / \mathrm{l}$ $\mathrm{VC}$ treatment for $24 \mathrm{~h}$ (VC group); and iii) $\mathrm{H}_{2} \mathrm{O}_{2}$ stimulation followed by passage 3 generation fetal placental mesenchymal stem cell (fPMSC) treatment for $24 \mathrm{~h}$ (supernatant group). In this experiment, stem cells were derived from human 


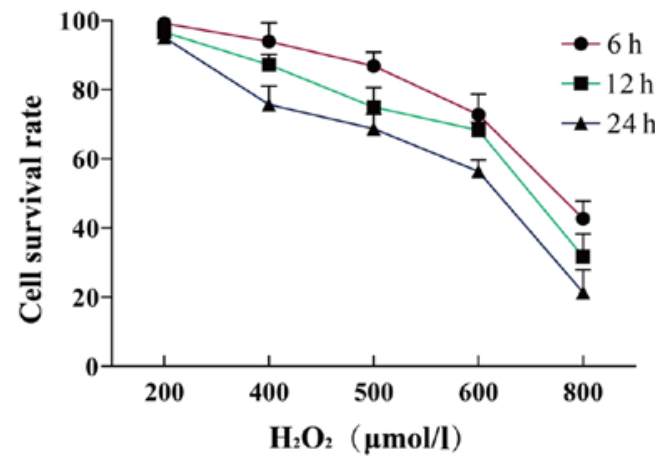

Figure 1. Effects of $\mathrm{H}_{2} \mathrm{O}_{2}$ treatment at varying concentrations and durations on $\mathrm{A} 549$ cell survival (mean \pm standard deviation; $\mathrm{n}=5$ ). When the concentration of $\mathrm{H}_{2} \mathrm{O}_{2}$ was $600 \mu \mathrm{mol} / \mathrm{l}$ and the time of stimulation was $24 \mathrm{~h}$, the cell survival rate was $56.41 \pm 3.31 \% . \mathrm{H}_{2} \mathrm{O}_{2}$, hydrogen peroxide.

placentas that were extracted from healthy parturients by cesarean section. Informed consent was obtained from the pregnant women and their families, and the experiments were approved by the Ethics Committee of the General Hospital of Ningxia Medical University (Yinchuan, Ningxia, China). FPMSCs were extracted and then preserved in liquid nitrogen. After the three groups were cultured, apoptosis was detected by Annexin V-fluorescein isothiocyanate/propidium iodide (FITC/PI) (Yantai Shuangshuang Chemical Co., Ltd., Shandong, Jinan, China) double staining, and the differences in apoptosis between the groups were detected by flow cytometry (BD Accuri ${ }^{\text {Tu }}$ C6, version 1.0.264.21; Accuri Cytometers, Inc., Ann Arbor, MI, USA).

Detection of apoptosis-associated protein and antioxidant signaling pathway-associated protein expression by western blot analysis. Protein was extracted by RIPA kit following the culture of the three groups, and the expression of the apoptosis-associated proteins BAX (eat. no. sc-7480; Santa Cruz Biotechnology, Inc.), Bcl-2 (cat. no. sc-7382; Santa Cruz Biotechnology, Inc.), Caspase-3 (cat. no. 9661; Cell Signaling Technology, Inc.), Nrf2 (cat. no. ab31163; Abcam) and Keap1
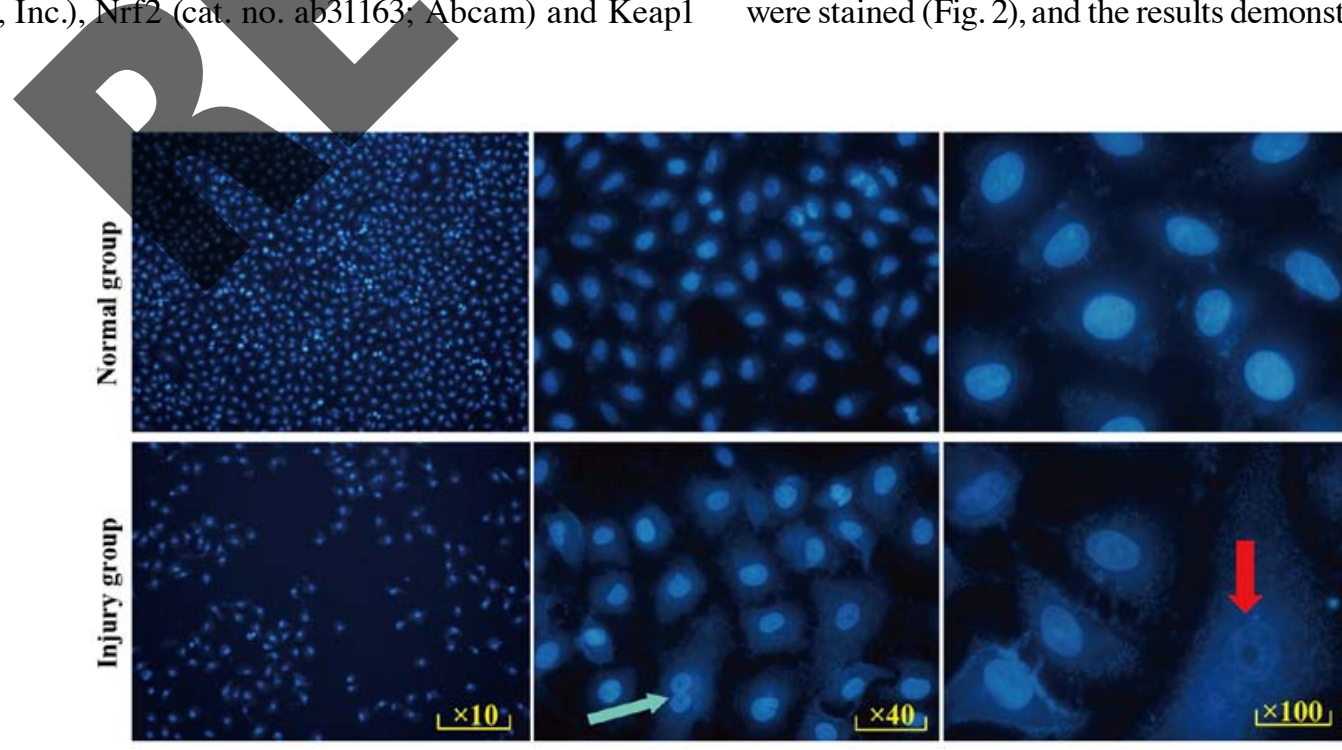

Figure 2. Hoechst 33258 staining in the normal and injury groups. The nuclei in the injury group presented with different degrees of pyknosis, fragmentation and lysis (red arrow). Compared with the normal group, granular blue fluorescence (green arrow) was visible in the nucleus. Marked apoptosis was observed in the injury group. 
Table I. Effects of $\mathrm{H}_{2} \mathrm{O}_{2}$ at different concentrations and for different treatment lengths on A549 survival (mean \pm standard deviation; $\mathrm{n}=5$ ).

\begin{tabular}{lccccc}
\hline & \multicolumn{5}{c}{$\mathrm{H}_{2} \mathrm{O}_{2}$ concentration, $\mu \mathrm{mol} / \mathrm{l}$} \\
\cline { 2 - 6 } Treatment length & 200 & 400 & 500 & 600 & 800 \\
$6 \mathrm{~h}$ & $99.23 \pm 7.07$ & $93.94 \pm 5.42$ & $86.87 \pm 4.04$ & $72.75 \pm 5.93$ & $42.68 \pm 5.07$ \\
$12 \mathrm{~h}$ & $96.59 \pm 1.56$ & $87.19 \pm 3.30$ & $74.98 \pm 5.65$ & $68.26 \pm 2.26$ & $31.68 \pm 6.57$ \\
$24 \mathrm{~h}$ & $95.09 \pm 5.86$ & $75.74 \pm 5.31$ & $68.71 \pm 4.19$ & $56.41 \pm 3.31$ & $21.39 \pm 6.52$ \\
\hline
\end{tabular}

$\mathrm{H}_{2} \mathrm{O}_{2}$, hydrogen peroxide.
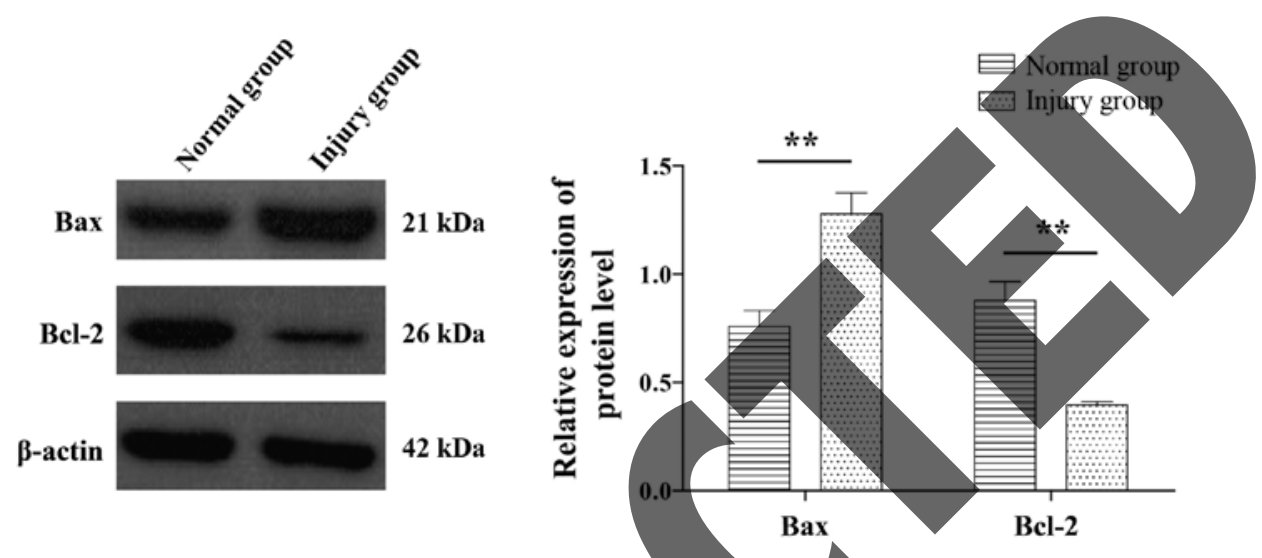

Figure 3. Western blot analysis to confirm model validity. Compared with that in the normal control group, the expression of the BAX gene in the injury group was increased, whereas the expression of the Bcl-2 gene was decreased. ${ }^{* *} \mathrm{P}<0.01$. BAX, apoptosis regulator BAX; Bcl-2, B-cell lymphoma 2.

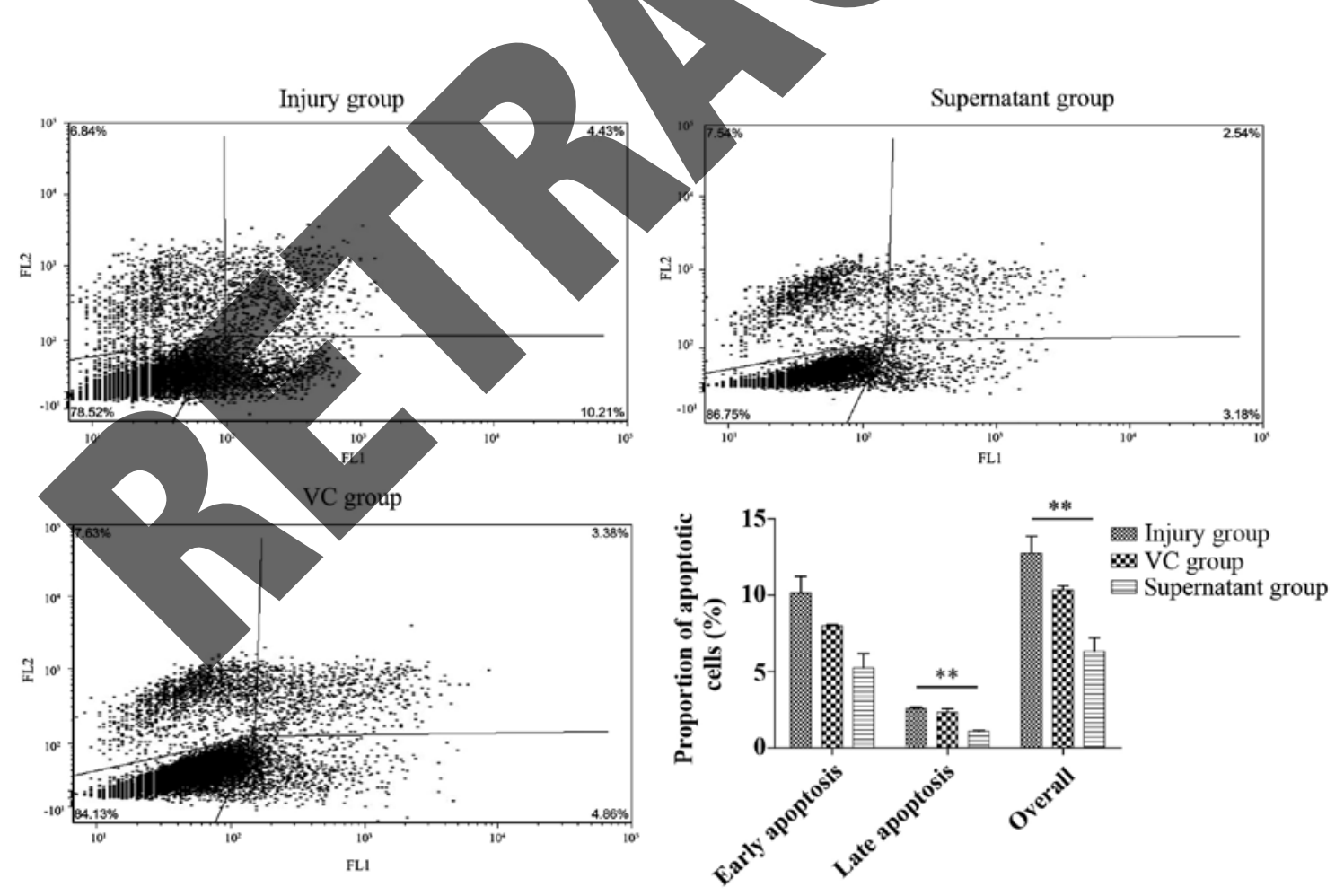

Figure 4. Apoptosis rate detected by flow cytometry. Apoptosis in the VC group and the supernatant group was significantly lower when compared with that in the hydrogen peroxide injury group. However, only late apoptosis and overall apoptosis rate in the supernatant group were significantly different from those in the injury group. ${ }^{* *} \mathrm{P}<0.01$. VC, vitamin $\mathrm{C}$.

in the injury group exhibited different degrees of pyknosis, fragmentation and lysis (red arrow). Compared with the normal group, granular blue fluorescence (green arrow) was visible in the nucleus. Marked apoptosis was observed in the injury group. 


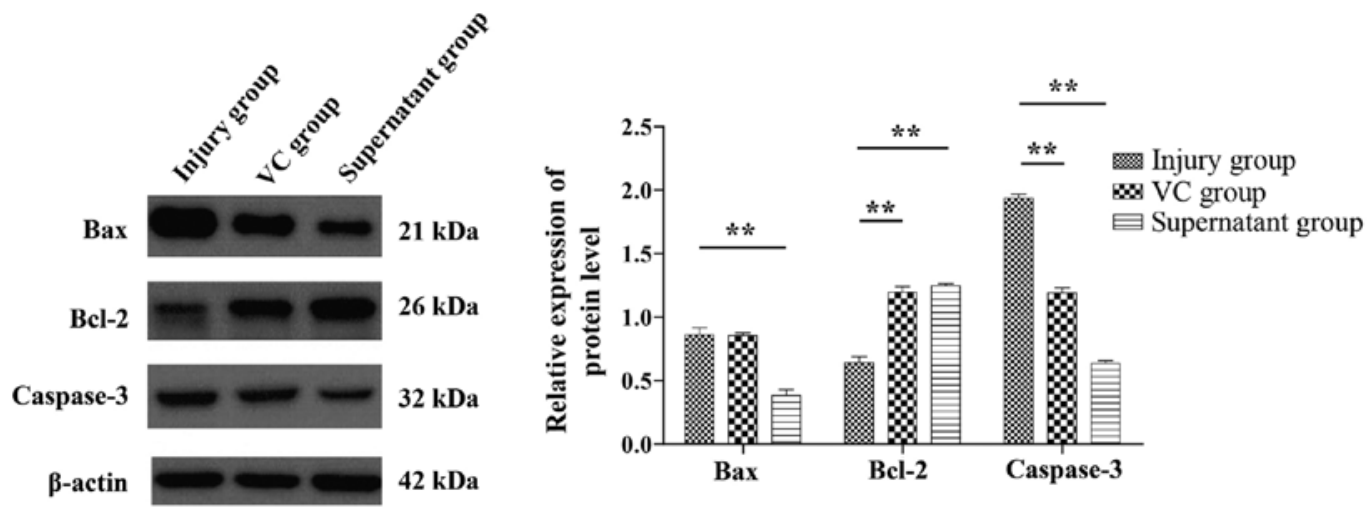

Figure 5. Expression of apoptosis-associated proteins as detected by western blot analysis. Compared with that in the injury group, the expression of BAX and Caspase- 3 was decreased in the VC and supernatant groups, whereas the expression of Bcl-2 was increased. ${ }^{* *} \mathrm{P}<0.01$. $\mathrm{VC}$, vitamin $\mathrm{C}$; BAX, apoptosis regulator BAX; Bcl-2, B-cell lymphoma 2.
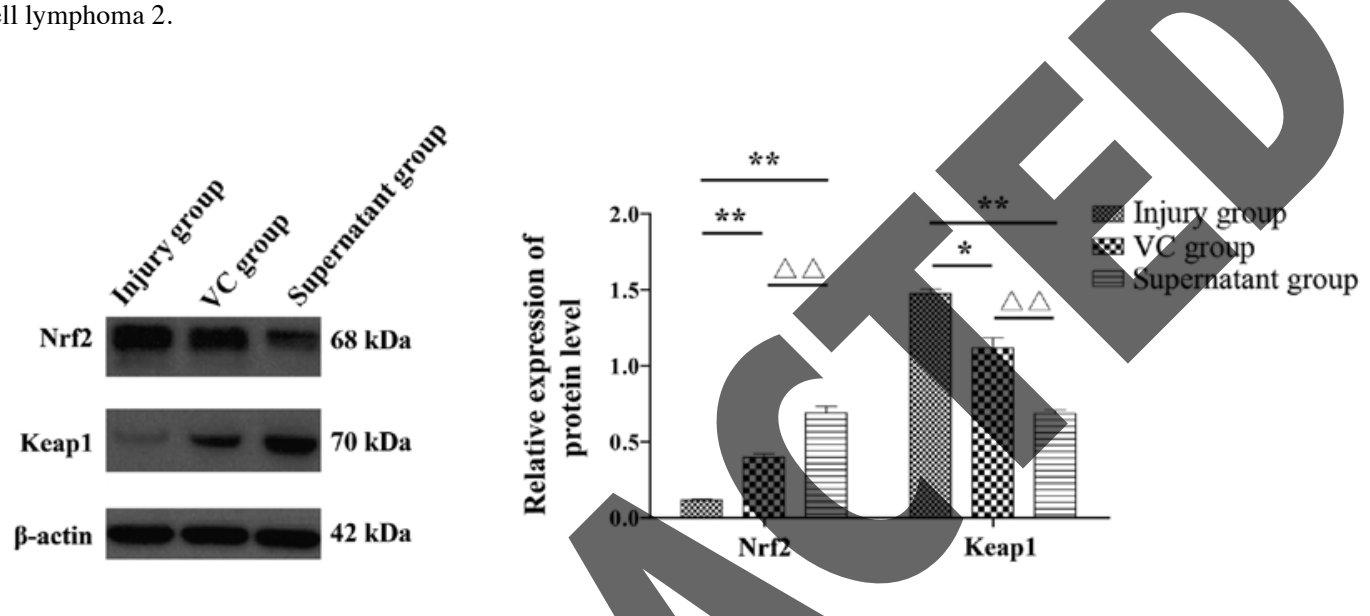

Figure 6. Western blot analysis of the expression of Nrf2-Keapl-ARE signaling pathway-associated proteins. Compared with that in the injury group, the level of Keap1 was decreased in the VC and supernatant groups, whereas the expression level of Nrf2 was increased. " $\mathrm{P}<0.05$ and ${ }^{* *} \mathrm{P}<0.01$ for the VC and supernatant groups vs. the injury group. ${ }^{\triangle} \mathrm{P}<0.01$ for the VC group vs. the supernatant groups. VC, vitamin C; Nrf2, nuclear factor erythroid 2-like 2; Keap1, kelch-like ECH-associated protein 1; ARE, antioxidant response element.

Validity of the model, as yerified by western blotting. $\mathrm{BAX}$ and $\mathrm{Bcl}-2$ are important apoptosis regulating genes. Compared with the normal control group, the injury group exhibited significantly increased expression of the BAX gene, whereas the expression of $\mathrm{Bcl}-2$ gene was significantly decreased $(\mathrm{P}<0.05$; Fig. 3 ).

Flow cytometry. Annexin V-FITC/PI double staining results (Fig. 4) demonstrated that the cell apoptosis in the VC and supernatant groups was significantly lower compared with that in $\mathrm{H}_{2} \mathrm{O}_{2}$ injury group. However, only late apoptosis and overall apoptosis rates in the supernatant group were significantly different from those in the injury group $(\mathrm{P}<0.05)$.

Expression of apoptosis-associated proteins. The apoptosis-associated proteins BAX, Bcl-2 and Caspase-3 were detected by western blot analysis (Fig. 5). Compared with that in the injury group, the expression of the BAX and Caspase-3 genes in the supernatant group was decreased, whereas the expression of Bcl-2 was increased. These differences were statistically significant $(\mathrm{P}<0.05)$.

Expression of antioxidant signaling pathway-associated proteins. The expression of Nrf2-Keap1-ARE signaling pathway-related proteins in the three groups was detected by western blot analysis (Fig. 6). The results revealed that compared with the injury group, the VC and supernatant groups exhibited a decreased level of Keap1 expression, whereas the expression level of Nrf2 was increased. These differences were also statistically significant $(\mathrm{P}<0.05)$.

\section{Discussion}

ALI is one of the most common diseases of the respiratory system. Due to the occurrence of acute alveolar inflammatory edema accompanied by rapid onset and a poor prognosis, ALI can rapidly develop into ARDS (16). Furthermore, the morbidity and mortality rates remain high. Therefore, a reliable and effective method of treatment for the disease is urgently required. Recently, multiple studies have demonstrated that ALI is associated with oxidative stress, and research into oxidative stress has gained increasing interest (17).

Oxidative stress is a result of an imbalance of oxidant and antioxidant substances, due to excessive ROS production by the organism, which results in oxidative damage to the tissues and cells. The lungs in particular are an organ sensitive to oxidative stress $(18,19)$. Therefore, preventing oxidative stress has become an important target for the treatment of lung diseases. The Nrf2-Keap1-ARE signaling pathway is recognized as a classic antioxidant pathway. Activation of 
the nuclear transcription factor $\mathrm{Nrf} 2$ promotes the expression of downstream molecular phase detoxification enzymes and antioxidant proteins, in order to improve antioxidant capacity (20). It has been demonstrated that $\mathrm{Nrf} 2$ activation may increase resistance to a series of diseases caused by oxidative stress, including central nervous system, cardiovascular, liver and kidney diseases, as well as tumors $(21,22)$. Therefore, the present study hypothesized that the protective effects of MSCs against the oxidative damage of lung epithelial cells may also be through this signaling pathway.

MSCs, as an ideal seed for tissue repair, have been widely recognized for their strong immune regulation and antioxidant capacity. Several studies have indicated that MSCs may be used to treat a variety of diseases $(23,24)$. In recent years, the use of MSCs has represented a breakthrough in the treatment of certain diseases, with promise shown for patients with refractory diseases in particular $(25,26)$. However, clinical application of MSCs may be problematic due to their potential tumorigenicity $(27,28)$. MSCs have low immunogenicity, but when in direct contact with the immune system, the risks and side effects should not be ignored, as a risk of tumor formation in MSCs has been previously reported in the literature $(29,30)$.

The initial step in the present study was to use $\mathrm{H}_{2} \mathrm{O}_{2}$ to model oxidative stress, and the cell survival rate, morphology and the expression of apoptosis-associated proteins were detected to ensure that the oxidative damage model of lung epithelial cells was successfully established for subsequent experiments. The experimental results demonstrated that, when lung epithelial cells were stimulated by $600 \mu \mathrm{mol} / 1 \mathrm{H}_{2} \mathrm{O}_{2}$. the cell survival rate was $56.41 \%$. Following Hoechst 33258 staining, marked apoptosis was observed in cells in the injury group. The integrity of the cytoplasm decreased, and nuclear fragmentation and nucleation occurred to different degrees. In addition, fluorescence staining in the nuclei was dense and compact. Western blot analysis demonstrated that compared with the normal group, the injury group exhibited increased expression of BAX, while $\mathrm{Bcl}-2$ expression was decreased, and the difference was statistically significant $(P<0.05)$. This confirmed that the model was successful.

Subsequently, the supernatant of fPMSCs was applied to lung epithelial cells following injury. Compared with the injury group, the supernatant group exhibited reduced cell apoptosis and apoptotic gene expression, and the expression of the antioxidant pathway key transcription factor $\mathrm{Nrf} 2$ increased. The antioxidant effects observed in the cells treated with fPMSC culture supernatant were higher compared with those in the $100 \mu \mathrm{mol} / \mathrm{l} \mathrm{VC}$ treated group. This suggested that fPMSC supernatant protected the lung epithelial cells against oxidative stress induced by $\mathrm{H}_{2} \mathrm{O}_{2}$, and this may have been via activation of the Nrf2-Keap1-ARE signaling pathway.

The detection of apoptotic genes and proteins associated with the Nrf2-Keap1-ARE signaling pathway by western blot analysis provided results consistent with expectations, and the differences were statistically significant $(\mathrm{P}<0.05)$. The positive effect of fPMSCs supernatant on the oxidative damage of lung epithelial cells was confirmed. During the process of Annexin V FITC/PI double staining to detect apoptosis using flow cytometry, it was determined that compared with that in the injury group, the apoptotic rate in the VC and supernatant groups was decreased, but only late apoptosis and the overall apoptotic rate in the supernatant group were significantly different from that in the injury group $(\mathrm{P}<0.05)$.

It was hypothesized that the lung epithelial cells damaged by $\mathrm{H}_{2} \mathrm{O}_{2}$ may have possessed a different proliferative capacity at the later stage due to the different degrees of apoptosis. Once the three cell groups were cultured, the dominant cells proliferated in large numbers, while the apoptotic cells did not proliferate or died. Therefore, in the detection of apoptotic cells by flow cytometry, the number of living cells accounted for a larger ratio, while the rate of apoptotic cells decreased, which may explain why the western blot analysis detected significant differences between the groups at the molecular level, whereas at the cellular level, the flow cytometry results were not always significantly different. This suggests that appropriate optimization of the experimental conditions is required for the next phase of research.

In summary, fPMSC culture supernatant was demonstrated to exhibit antioxidant ability, and to a certain extent, to reduce the apoptosis induced by $\mathrm{H}_{2} \mathrm{O}_{2}$, at least in part via activation of the Nrf2-Keap1-ARE signaling pathway. This suggested that for the clinical application of MSCs, in addition to the cells themselves, the culture supernatant may also have potential therapeutic value. In addition to its antioxidative effects, the supernatant of MSCs may have other beneficial functions, which require further investigation.

\section{Acknowledgements}

The authors would like to express their sincere thanks to Spandidos Publications Ltd. for the English language revisions in this manuscript.

Funding

The present study was supported by the Youth Medical Talent of Jiangsu Province (grant no. QNRC2016475) and the Science and Technology Commission of Yancheng City (grant no. YK2015002).

\section{Availability of data and materials}

The datasets used and/or analyzed during the current study are available from the corresponding author on reasonable request.

\section{Authors' contributions}

JW and AS contributed to study concept and design, acquisition of data, analysis and interpretation of data, and drafting of the manuscript; CC contributed to the statistical analysis; WW contributed to the study concept, study supervision and critical revision of the manuscript; $\mathrm{CX}$ and $\mathrm{NG}$ contributed to the study concept and design, study supervision and critical revision of the manuscript. All authors read and approved the final manuscript.

\section{Ethics approval and consent to participate}

All procedures performed involving human participants were in accordance with the ethical standards of the Ethics Committee of the General Hospital of Ningxia Medical 
University (Yinchuan, Ningxia, China), and the First People's Hospital of Yancheng City (Yancheng, Jiangsu, China), and with the 1964 Helsinki declaration and its later amendments or comparable ethical standards. Informed consent was obtained from all participants enrolled in the study. From all participants, tissue samples were included in the sample pool and informed consent was collected from all participants prior to storage of the sample. An ethical review of the sample library has been submitted and the review contains the informed consent of all participants.

\section{Patient consent for publication}

Not applicable.

\section{Competing interests}

The authors declare that they have no competing interests.

\section{References}

1. Jin Z, Chun Suen K and Ma D: Perioperative 'remote' acute lung injury: Recent update. J Biomed Res 31: 197-212, 2017.

2. Hayes M, Curley G, Ansari B and Laffey JG: Clinical review: Stem cell therapies for acute lung injury/acute respiratory distress syndrome - hope or hype? Crit Care 16: 205, 2012.

3. Han F, Luo Y, Li Y, Liu Z, Xu D, Jin F and Li Z: Seawater induces apoptosis in alveolar epithelial cells via the Fas/FasL-mediated pathway. Respir Physiol Neurobiol 182: 71-80, 2012.

4. Al-Biltagi MA, Abo-Elezz AA, Elshafiey RM, Suliman Mabrouk MM and Mourad HA: The predictive value of solut endothelial selectin plasma levels in children with acute lu injury. J Crit Care 32: 31-35, 2016.

5. Lima Trajano ET, Sternberg C, Caetano M, S Porto LC, Santos JC, Ribeiro ML, Magalhães Benjamim CF, et al: Endotoxin-induced dependent upon oxidative response. Inhal 2011.

6. Poljsak B, Suput D and Milisav I. Ac ROS and antioxidants: when to $y$ Oxid Med Cell Longev 2013: 95

7. Yang $C$, Jiang J, Yang $X$, Wang $H$ and Du J. Stem/progenitor cells in endogenous repairing responses. New toolbox for the treatment of acute lung injury J Transl Med 14: 47, 2016.

8. Miller DM, Singh IN, Wang JA and Hall ED: Administration of the Nrf2-ARE activators sulforaphane and carnosic acid attenu ates 4-hydroxy-2-nonenal-induced mitochondrial dysfunction ex vivo. Free Radic Biol Med 57: 1-9, 2013

9. Suniara RK, Jenkinson EJ and Owen JJ: An essential role for thymic mesenchyme in early T cell development. J Exp Med 191: 1051-1056, 2000

10. Shalaby SM, El-Shal AS, Abd-Allah SH, Selim AO, Selim SA, Gouda ZA, Abd El Motteleb DM, Zanfaly HE, El-Assar HM and Abdelazim S: Mesenchymal stromal cell injection protects against oxidative stress in Escherichia coli-induced acute lung injury in mice. Cytotherapy 16: 764-775, 2014.

11. Wilson JG, Liu KD, Zhuo H, Caballero L, McMillan M, Fang X, Cosgrove K, Vojnik R, Calfee CS, Lee JW, et al: Mesenchymal stem (stromal) cells for treatment of ARDS: A phase 1 clinical trial. Lancet Respir Med 3: 24-32, 2015.

12. Castro-Manrreza ME and Montesinos JJ: Immunoregulation by mesenchymal stem cells: Biological aspects and clinical applications. J Immunol Res 2015: 394917, 2015.
13. Zhang G, Zou X, Huang Y, Wang F, Miao S, Liu G, Chen M and Zhu Y: Mesenchymal stromal cell-derived extracellular vesicles protect against acute kidney injury through anti-oxidation by enhancing Nrf2/ARE activation in Rats. Kidney Blood Press Res 41: 119-128, 2016.

14. Pleyer L, Valent P and Greil R: Mesenchymal stem and progenitor cells in normal and dysplastic hematopoiesis-masters of survival and clonality? Int J Mol Sci 17: 1009, 2016

15. Lv H, Liu Q, Wen Z, Feng H, Deng X and Ci X: Xanthohumol ameliorates lipopolysaccharide (LPS)-induced acute lung injury via induction of AMPK/GSK3 $\beta$-Nrf2 signal axis. Redox Biol 12: 311-324, 2017.

16. Ware LB and Matthay MA: The acute respiratory distress syndrome. N Engl J Med 141: 460-470, 2000.

17. Keum YS and Choi BY: Molecular and chemical regulation of the Keap1-Nrf2 signaling pathway. Molecules 19: 10074-10089, 2014.

18. Wang Z, Ji C, Wu L, Qiu J, Li Q, Shao Z and Chen G: Tertbutylhydroquinone alleviates early brain injury and cognitive dysfunction after experimental subarachnoid hemorrhage: Role of Keap1/Nrf2/ARE pathway. PLoS Qne 9: e97685, 2014.

19. Liu X, Zheng P, Xiaodong G, Guanghui D, Hongbin CH, Zan ZH, Rongrong $\mathrm{H}$, Xinxin N, Jing $\mathrm{SH}$ and Yihua A: A preliminary evaluation of efficacy and safety of Wharton's jelly mesenchymal stem cell transplantation in patients with type 2 diabetes mellitus. Stem Cell Research \& Therapy 5: 1-

20. Jang YO, Kim YJ, Baik SK, Kim MY, Eon YW, Cho MY, Park HJ, Park SY, Kim BR, Kim JW et al: Histological improvement following administration of autologous bone marrow-derived mesenchymal stem cells for alcoholic cirrhosis: A pilot study: Liverint 34: 33-41, 2014

21. Hare JM, Fishman JE, Gerstenblith G, DiFede Velazquez DL, Zambrano JP, Suncion VY, Tracy M, Ghersin E, Johnston PV, Brinker JA, Breton E, et al: Comparison of allogeneic vs autologous bone marrow-derived mesenchymal stem cells delivered by transendocardial injection in patients with ischemic cardiomyopathy: JAMA 308: 2369-2379, 2012.

2. Ni S, Wang D, Qiu X, Pang L, Song Z and Guo K: Bone marrow mesenchymal stem cells protect against bleomycin-induced pulmonary fibrosis in rat by activating Nrf 2 signaling. Int J Clin Exp Pathol 8: 7752-7761, 2015.

. Chang YS, Ahn SY, Yoo HS, Sung SI, Choi SJ, Oh WI and Park WS: Mesenchymal stem cells for bronchopulmonary dysplasia: phase 1 dose-escalation clinical trial. J Pediatr 164: 966-972, 2014.

24. Haddad R and Saldanha-Araujo F: Mechanisms of T-cell immunosuppression by mesenchymal stromal cells: What do we know so far? BioMed Res Int 2014: 216806, 2014.

25. Martinet L, Fleury-Cappellesso S, Gadelorge M, Dietrich G, Bourin P, Fournié JJ and Poupot R: A regulatory cross-talk between $\mathrm{V} \gamma 9 \mathrm{~V} \delta 2 \mathrm{~T}$ lymphocytes and mesenchymal stem cells. Eur J Immunol 39: 752-762, 2009.

26. Tasso R, Augello A, Carida' M, Postiglione F, Tibiletti MG, Bernasconi B, Astigiano S, Fais F, Truini M, Cancedda R, et al: Development of sarcomas in mice implanted with mesenchymal stem cells seeded onto bioscaffolds. Carcinogenesis 30: 150-157, 2009.

27. Chen W, Huang Y, Han J, Yu L, Li Y, Lu Z, Li H, Liu Z, Shi C, Duan F, et al: Immunomodulatory effects of mesenchymal stromal cells-derived exosome. Immunol Res 64: 831-840, 2016.

28. Sabin K and Kikyo N: Microvesicles as mediators of tissue regeneration. Transl Res 163: 286-295, 2014.

29. Sdrimas K, Kourembanas S: MSC microvesicles for the treatment of lung disease: a new paradigm for cell-free therapy. Antioxid Redox Signal 21: 1905-1915, 2014.

30. Hashemian SJ, Kouhnavard $M$ and Nasli-Esfahani E: Mesenchymal stem cells: Rising concerns over their application in treatment of type one diabetes mellitus. J Diabetes Res 2015: 675103, 2015. 